\title{
A modified method for purification of Eimeria tenella sporozoites
}

\author{
Zaida Rentería-Solís $^{1,2}$ (D) $\cdot$ Runhui Zhang ${ }^{1} \cdot$ Shahinaz Taha ${ }^{1} \cdot$ Arwid Daugschies ${ }^{1,2}$
}

Received: 30 October 2019 / Accepted: 6 January 2020 / Published online: 16 January 2020

(C) The Author(s) 2020

\begin{abstract}
Coccidiosis is an economically important gastrointestinal disease in domestic fowl. Eimeria species are the causative agents of avian coccidiosis. Current challenges in management and prevention of eimeriosis enhance the need for research in this field. Sporozoite purification is a necessary step for Eimeria spp. in vitro infection models. Current alternatives such as DE-52 anion exchange chromatography and Percoll gradient require time and resources. We present a modified protocol consisting on vacuum filtration of sporozoites using a disposable 5- $\mu \mathrm{L}$ filter. Yield percentages were similar to those reported for Percoll gradient purification. By reducing time and efforts during sporozoite purification, it could be possible to increase resources in other areas of Eimeria studies.
\end{abstract}

Keywords Eimeria $\cdot$ Coccidiosis $\cdot$ Chicken $\cdot$ Sporozoite $\cdot$ Purification $\cdot$ Excystation

\section{Introduction}

Coccidiosis is one of the most important diseases of the poultry industry. It can cause economic losses of over 800 million US dollars annually worldwide (Shirley et al. 2007). Eimeria spp. are the cause of coccidiosis in domestic fowl. These parasites cause gastrointestinal problems characterised by diarrhoea, weight loss, reduced egg production and in some cases death. In chickens, seven Eimeria spp. are commonly found in the field. They differentiate themselves in oocyst morphology, virulence and area of infection in the digestive system. Additionally, infection with Eimeria spp. can promote opportunistic infections by other pathogens like bacteria (Collier et al. 2008). Eimeria oocysts are highly tolerant to the environment, which makes control measures difficult. Additionally, the common prophylactic use of anticoccidial feed additives has led to wide spread anticoccidial resistance

Zaida Rentería-Solís and Runhui Zhang contributed equally to this work.

Section Editor: David S. Lindsay

Zaida Rentería-Solís

zaida_melina.renteria_solis@vetmed.uni-leipzig.de

1 Institute for Parasitology, Faculty of Veterinary Medicine, Centre for Infectious Diseases, University of Leipzig, An den Tierkliniken 35, 04103 Leipzig, Germany

2 Albrecht-Daniel-Thaer-Institute, An den Tierkliniken 29, 04103 Leipzig, Germany
(Stephan et al. 1997). Eimeria tenella is one of the most frequent and pathogenic Eimeria spp. in domestic fowl (Blake et al. 2015). It can cause haemorrhagic caecal disease with fatal outcome in some cases. Given its importance, E. tenella is commonly studied and used as a model for chicken coccidiosis (Blake et al. 2015, Hiob et al. 2017, Thabet et al. 2017, Zhang et al. 2018). Nevertheless, there is still a large need for more research within this field. A valuable tool regularly used as an alternative to in vivo model is in vitro infection assay (Dimier-Poisson et al. 2004, Thabet et al. 2017). Purification of Eimeria sporozoites is an important step before cell culture infection. There are currently some purification methods available (Schmatz et al. 1984, Dulski and Turner 1988, Zhang et al. 2015). Purification of E. tenella sporozoites by DE-52 anion exchange chromatography is a method regularly used during in vitro studies (Schmatz et al. 1984). Nevertheless, this technique involves time and additional materials. Similarly, the use of Percoll gradients also requires time for gradient preparation (Dulski and Turner 1988). In this study, we present a simple alternative for E. tenella sporozoite purification.

\section{Material and methods}

Eimeria tenella Houghton strain (kindly provided by Prof. Dr. D. P. Blake, Royal Veterinary College, University of London, UK) was passaged in healthy 10-day old chickens. Oocysts 
were purified from the faeces of the chickens following a modified protocol (Eckert et al. 1995). Briefly, faecal samples were collected and transferred to a 5-L plastic bucket. Thereafter, 2 volumes of tap water were added. Then, samples were homogenised with a hand blender (Braun, Frankfurt, Germany) until the mixture was homogeneous. The homogenate was filtered through a $250-\mu \mathrm{m}$-pore-size sieve and the filtrate transferred to a 2-L cylinder and sedimented overnight. Afterwards, the supernatant was discarded, and the sediment was resuspended in saturated saline solution. Followed by centrifugation of the resuspension at $1300 \times \mathrm{g}$ for $10 \mathrm{~min}$. Afterwards, suspended oocysts were collected and washed with tap water by centrifugation at $1300 \times g$ for $10 \mathrm{~min}$. Finally, purified oocysts were collected and incubated for sporulation in $4 \%$ potassium dichromate solution at room temperature for $48 \mathrm{~h}$. Sporulated oocysts were stored at $4{ }^{\circ} \mathrm{C}$ until further use.

The entire protocol was performed in triplicate with a total amount of $1.5 \times 10^{5}$ oocysts per replicate. Before excystation, sporulated oocysts were cleaned from the potassium dichromate through centrifugation. Briefly, oocysts were centrifuged at $1300 \times \mathrm{g}$ for $10 \mathrm{~min}$ at room temperature (RT). Afterwards, the supernatant was discarded and the pellet was resuspended in sterile PBS ( $\mathrm{pH} 7.0$ ) and centrifuged again at $1300 \times \mathrm{g}$ for $10 \mathrm{~min}$ at RT. This centrifugation step was repeated twice or until the supernatant was clear. Oocysts' surface sterilisation was performed as follows: after the last centrifugation of the previous step, the pellet was resuspended in $12 \%$ sodium hypochlorite (Carl-Roth, Karlsruhe, Germany) and incubated in a tube mixer at room temperature for $10 \mathrm{~min}$. Immediately afterwards, the oocysts were centrifuged at $2500 \times \mathrm{g}$ for $3 \mathrm{~min}$. After centrifugation, the white cloudy layer formed at the top of the supernatant was transferred to clean $50-\mathrm{mL}$ tube and resuspended in sterile PBS ( $\mathrm{pH} 7.0)$. Oocysts were cleaned from sodium hypochlorite through vacuum filtration using a sterile1- $\mu \mathrm{m}$ Pluristrainer ${ }^{\circledR}$ filter (Pluriselect, Leipzig, Germany) mounted on a sterile connector ring (Pluriselect, Leipzig, Germany). After filtration, the filter was washed 3 times with sterile PBS ( $\mathrm{pH} 7.0$ ) in order to recover the oocysts. The filtration step was repeated once more. After the second filtration, recovery of oocysts was done using sterile PBS with $\mathrm{pH}$ 7.6-8.0. Alternatively, the oocysts can also be cleaned from the sodium hypochlorite by centrifugation at $1300 \mathrm{~g}$ for $10 \mathrm{~min}$, followed by 3 wash cycles with PBS at $1300 \mathrm{~g}$ for $10 \mathrm{~min}$. The recovered oocysts were immediately transferred to a $15-\mathrm{mL}$ falcon tube with $0.5-\mathrm{mm}$ sterilised glass beads (Carl Roth, Karlsruhe, Germany). The glass beads filled the tube up to the 0.5 - to $1-\mathrm{mL}$ mark. Once added to the tube with glass beads, the oocyst suspension was filled with sterile PBS (pH 7.6-8.0) up to $4 \mathrm{~mL}$. Release of the oocysts' wall was performed by vortexing the tubes for 3 cycles of $20 \mathrm{~s}$. After each $20 \mathrm{~s}$ cycle, the sporocyst-oocyst ratio was examined using a light microscope. If necessary, an additional 20 -s cycle was performed in order to maximise the number of released sporocysts. Afterwards, the supernatant with released sporocysts was transferred to a $15-\mathrm{mL}$ falcon tube. The glass beads were washed 2-3 times with $3 \mathrm{~mL}$ of sterile PBS (pH 7.6-8.0) to collect remaining sporocysts. Later on, sporocysts were centrifuged at $2500 \times \mathrm{g}$ for $10 \mathrm{~min}$ at RT. The supernatant was discarded and the pelleted sporocysts were enzymatically excysted. Briefly, sporocysts were incubated with $0.25 \%$ trypsin (Biochrom AG, Berlin, Germany) and $4 \%$ sodium taurocholic acid (Sigma, Taufkirchen, Germany) in sterile PBS (pH 7.6-8.0) at $41^{\circ} \mathrm{C}$ for 60 to $90 \mathrm{~min}$. Monitoring of excystation rate was performed every $30 \mathrm{~min}$ with light microscopy. After incubation, free sporozoites were transferred to $50 \mathrm{~mL}$ of $1 \%$ glucose (Carl Roth $\mathrm{GmbH}$, Karsruhe, Germany) in sterile PBS ( $\mathrm{pH}$ 7.0) previously warmed to $41^{\circ} \mathrm{C}$. Immediately afterwards, sporozoites were purified by vacuum filtration, using a $5-\mu \mathrm{m}$ Pluristrainer ${ }^{\circledR}$ filter (Pluriselect, Leipzig, Germany) mounted on a sterile connector ring (Pluriselect, Leipzig, Germany). To reduce the amount of sporocyst residue, sporozoites can also be filtered by gravity. For this option, the sterile connector ring is superfluous. After filtration, sporozoites are washed from the glucose solution by centrifugation at $3200 \times \mathrm{g}$ for $10 \mathrm{~min}$ at RT. Right thereafter, the supernatant is carefully removed and the sporozoites are resuspended in the appropriate infection medium. Finally, sporozoites are counted under a light microscope using a Neubauer chamber (depth $0.100 \mathrm{~mm}$, Paul Marienfeld GmbH, Lauda-Königshofen, Germany).

Sporozoite viability after purification was assessed according to Thabet et al. (2015). Briefly, Madin-Darby Bovine Kidney Cells (MDBK) were seeded in 24-well plates with Dulbecco's Modified Eagle's Medium (DMEM) with 5\% foetal bovine serum (FBS), $100 \mathrm{IU}$ penicillin, $100 \mu \mathrm{g} / \mathrm{mL}$ streptomycin and $2.5 \mu \mathrm{g} / \mathrm{mL}$ amphotericin B. Cells were incubated at $37{ }^{\circ} \mathrm{C}$ and $5 \% \mathrm{CO}_{2}$ until they reached 80 to $90 \%$ confluence. Cells were infected with freshly purified E. tenella sporozoites $\left(5 \times 10^{4} /\right.$ well). The negative control group $(\mathrm{NC})$ consisted of uninfected MDBK cells. All groups were performed in triplicate. Following infection, all groups of cell cultures were incubated at $41{ }^{\circ} \mathrm{C}$ and $5 \% \mathrm{CO}_{2}$ for $24 \mathrm{~h}$. After $24 \mathrm{~h}$, cells were washed 3 times with sterile PBS (pH 7.0) and fresh DMEM was added. After incubation at $41{ }^{\circ} \mathrm{C}$ for $96 \mathrm{~h}$ DNA was extracted from the cells using the DNeasy Blood \& Tissue kit (Qiagen, Hilden, Germany) following manufacturer's instructions. Quantification of E. tenella genomic copies was performed in triplicate using a real-time PCR assay. Additionally a non-template control (NTC) consisting of nuclease-free water was added in triplicate to the assay. The primers ETF 5'-TGGAGGGGATTATG AGAGGA-3' and ETR 5'-CAAGCAGCATGTAA CGGAGA-3' were used to amplify a 147-bp fragment of the E. tenella internal transcribed spacer 1 (ITS-1) gene using a SYBR Green-based assay (Thermo Scientific, Darmstadt, 
Germany) according to Kawahara et al. (2008). Finally, the number of gene copies was calculated from the qPCR data by using a standard curve prepared from a serial dilution of cloned ITS-1 gene fragment according to Thabet et al. (2015).

\section{Results and discussion}

Sporozoites recovered using the described protocol were clean and motile. After oocysts sterilisation with $12 \%$ sodium hypochlorite, yield efficacy was calculated for every further step of the procedure (Table 1). Overall, a yield of $35.69 \pm 8.93 \%$ was recorded. Parasite loss was highest after grinding of oocysts with a yield mean of $55.55 \pm 9.17 \%$ (Table 1). A low loss of sporozoites was calculated for the purification step with a mean value of $3.33 \%$. Sporozoites collected by the current procedure were viable as assessed by successful in vitro cell invasion and replication. An initial number of $1.21 \pm 0.39 \times$ $10^{5}$ gene copies of the selected E. tenella gene fragment was determined by qPCR in $3 \mu \mathrm{L}$ of total DNA after $96 \mathrm{~h}$ of incubation of MDBK following infection with $4 \times 10^{4}$ sporozoites.

We present a modified protocol for E. tenella sporozoite purification. An earlier version of this protocol has been successfully implemented in in vitro studies (Zhang et al. unpublished data, Rentería-Solís et al. unpublished data). Purification of E. tenella sporozoites can also successfully be performed by other protocols such as DE-52 anion exchange chromatography (Schmarz et al. 1984), Percoll gradients (Dulski an Turner 1988, Thabet et al. 2015) or with a 1400-mesh filter (Zhang et al. 2015). The protocol described in this report uses a plastic disposable $5-\mu \mathrm{m}$ filter that perfectly fits to a $50-\mathrm{mL}$ falcon tube. In this study, the Pluristrainer ${ }^{\circledR}$ model from Pluriselect (Leipzig, Germany) was used; however, similar products by other manufacturers exist in the market and are probably equally suited. By using a disposable $5-\mu \mathrm{L}$ filter, there is no need of applying additional purification steps. This reduces the costs and time spent on sporozoite purification significantly thus saving resources in laboratory approaches.
Dulski and Turner (1988) reported a total yield of 39\% of sporozoites using Percoll gradient for purification. Similar efficacy was obtained with the method presented in this study with $35.69 \pm 8.93 \%$ of sporozoites recovered. Interestingly, Schmatz et al. (1984) reported a recovery of between 94 and $100 \%$ sporozoites after DE-52 anion exchange chromatography. However, the authors do not specify if the recovery percentage is calculated based on the initial amount of oocysts or in relation to the number of sporozoites collected during the previous step of purification. In our study, only $55 \pm 9.17 \%$ of sporocysts were recovered after grinding of the oocysts' wall with glass beads. Similar results have been reported by Dulski and Turner (1988) with 51\% of recovered sporocysts. Mechanical grinding with glass beads is not likely to destroy every single oocyst's wall. Excess of mechanical impact bears the risk of destruction of already liberated sporocysts and sporozoites. Alternatively, a mortar and pestle can also be used to grind oocysts instead of glass beads. Doran and Farr (1962) report a recovery of 30-65\% of available E. acervulina sporocysts using the mortar method. Furthermore, in our new protocol, an average of only $3.33 \%$ of excysted sporozoites was lost during purification after sporocysts excystation. These results could be similar to those reported by Schmatz et al. (6 to $0 \%$ loss) if their recovery percentage was calculated from the total of excysted sporozoites. Dulski and Turner (1988) described only $1 \%$ loss of sporozoites after Percoll purification. However, that method comprises two Percoll gradient centrifugations of $20 \mathrm{~min}$ each which is time-consuming.

Research on chicken Eimeria spp. brings insights into coccidian metabolism, genetics, epidemiology and hostparasite interaction (Györke et al. 2013, Blake et al. 2015, Zhang et al. 2015). These developments could translate into improved therapies or preventive measurements. As ethically responsible study designs, in vitro models are pivotal to fulfil the goals of the 3R principle in animal research (Russell and Burch 1959). Eimeria sporozoites are commonly used in in vitro studies (Zhou et al. 2013, Thabet et al. 2015, Zhang et al. 2015, Bussière et al. 2018, Zhang et al. 2018). Sporozoites are the first cell invasive stage during eimeriosis. Therefore, any research on in vitro features of these coccidia depends on availability of viable sporozoites.

Table 1 Parasite recovery and yield efficacy

\begin{tabular}{lllr}
\hline Step & Recovered parasitic stage & Quantity of parasites after each step $^{\mathrm{a}}$ & ${\text { Yield }(\%)^{\mathrm{a}, \mathrm{b}}}^{\mathrm{a}}$ \\
\hline Cleaning of oocysts with 1- $\mu \mathrm{m}$ filter & Oocysts & $1.19 \pm 0.13 \times 10^{5}$ & $79.44 \pm 9.17$ \\
Oocysts mechanical grind with glass beads & Sporocysts & $3.33 \pm 0.55 \times 10^{5}$ & $55.55 \pm 9.17$ \\
Excystation & Sporozoites & $4.46 \pm 0.9 \times 10^{5}$ & $39.02 \pm 7.50$ \\
Sporozoites purification with 5- $\mu \mathrm{m}$ filter & Sporozoites & $4.28 \pm 1.07 \times 10^{5}$ & $35.69 \pm 8.93$ \\
\hline
\end{tabular}

${ }^{\text {a }}$ Quantities and yields reported are the means \pm standard deviations of triplicate

${ }^{\mathrm{b}}$ Yield percentages calculated from an initial amount of $1.5 \times 10^{5}$ oocysts

${ }^{\mathrm{c}}$ Yield percentages of earlier steps during oocysts cleaning were not calculated 
The development of a time saving and economic alternative to current methods for sporozoite purification could increase the interest in eimeriosis research. Furthermore, this protocol was established in E. tenella. Therefore, applications of this modified method in further Eimeria species should be encouraged and likewise reported.

Acknowledgements The authors are grateful to M. Fritsche and R. Schumacher (Institute for Parasitology, Faculty of Veterinary Medicine, University of Leipzig) for their excellent assistance as animal keepers.

Funding information Open Access funding provided by Projekt DEAL. This project was partially funded by the Flexible Fonds Program for junior scientists of the University of Leipzig (project number: 43700871). We thank the Karl-Enigk-Stiftung for the scholarship of R. Zhang, as well as the Sudanese Ministry of Higher Education and Scientific Research for providing finance assistance to $\mathrm{S}$. Taha.

\section{Compliance with ethical standards}

Ethical approval All procedures performed involving animals were in accordance with the ethical standards of the local authorities (Landesdirektion Sachsen, permit no. A04/19).

Conflict of interest The authors declare that they have no conflict of interest.

Open Access This article is licensed under a Creative Commons Attribution 4.0 International License, which permits use, sharing, adaptation, distribution and reproduction in any medium or format, as long as you give appropriate credit to the original author(s) and the source, provide a link to the Creative Commons licence, and indicate if changes were made. The images or other third party material in this article are included in the article's Creative Commons licence, unless indicated otherwise in a credit line to the material. If material is not included in the article's Creative Commons licence and your intended use is not permitted by statutory regulation or exceeds the permitted use, you will need to obtain permission directly from the copyright holder. To view a copy of this licence, visit http://creativecommons.org/licenses/by/4.0/.

\section{References}

Blake DP, Clark EL, Macdonald SE, Thenmozhi V, Kundu K et al (2015) Population, genetic, and antigenic diversity of the apicomplexan Eimeria tenella and their relevance to vaccine development. PNAS:E5343-E5350

Bussière FI, Niepceron A, Sausset A, Esnault E, Silvestre A, Walker RA, Smith NC, Quére P, Laurent F (2018) Establishment of an in vitro chicken epithelial cell line model to investigate Eimeria tenella gamete development. Parasit Vectors 11:44. https://doi.org/10. 1186/s13071-018-2622-1

Collier CT, Hofacre CL, Payne AM, Anderson DB, Kaiser P, Mackie RI, Gaskins HR (2008) Coccidia-induced mucogenesis promotes the onset of necrotic enteritis by supporting Clostridium perfringens growth. Vet Immunol Immunopathol 122:104-115

Dimier-Poisson IH, Bout DT, Quére P (2004) Chicken primary enterocytes: inhibition of Eimeria tenella replication after activation with crude interferon-gama supernatants. Avian Dis 48:617-627

Doran DJ, Farr MM (1962) Excystation of the poultry coccidium, Eimeria acervulina. J Protozool 9:154-161

Dulski P, Turner M (1988) The purification of sporocysts and sporozoites from Eimeria tenella oocysts using Percoll density gradients. Avian Dis 32:235-239

Eckert J, Braun R, Shirley MW, Coudert P (1995) Biotechnology guidelines on techniques in coccidiosis research. COST European Cooperation in the field of Scientific and Technical Research, Brussels, Belgium

Györke A, Pop L, Cozma V (2013) Prevalence and distribution of Eimeria species in broiler chicken farms of different capacities. Parasite 20:50

Kawahara F, Taira K, Nagai S, Onaga H, Onuma M, Nunoya T (2008) Detection of five avian Eimeria species by species-specific real-time polymerase chain reaction assay. Avian Dis 52:652-656

Russell WMS, Burch RL (1959) The principles of humane experimental technique. Methuen, London

Shirley MW, Smith AL, Blake DP (2007) Challenges in the successful control of avian coccidia. Vaccine 25:5540-5547

Schmatz DM, Crane MST, Murray PK (1984) Purification of Eimeria sporozoites by DE-52 anion exchange chromatography. J Protozool 31:181-183

Thabet A, Alnassan AA, Daugschies A, Bangoura B (2015) Combination of cell culture and qPCR to assess the efficacy of different anticoccidials on Eimeria tenella sporozoites. Parasitol Res 114: 2155-2163

Thabet A, Zhang R, Alnassan AA, Daugschies A, Bangoura B (2017) Anticoccidial efficacy testing: in vitro Eimeria tenella assays as replacement for animal experiments. Vet Parasitol 233:86-96. https://oi.org/10.1016/j.vetpar.2016.12.005

Zhang R, Thabet A, Hiob L, Zheng W, Daugschies A, Bangoura B (2018) Mutual interactions of the apicomplexan parasites Toxoplasma gondii and Eimeria tenella with cultured poultry macrophages. Parasit Vectors 11:453. https://doi.org/10.1186/s13071-018-3040-0

Zhang Y, Zheng MX, Xu ZY, Xu HC, Cui XZ, Yang SS, Zhao WL, Li S, Lv QH, Bai R (2015) Relationship between Eimeria tenella development and host cell apoptosis in chickens. Poult Sci 94:2970 2979. https://doi.org/10.3382/ps/pev293

Zhou Z, Wang Z, Cao L, Hu S, Zhang Z, Qin B, Guo Z, Nie K (2013) Upregulation of chicken TLR4, TLR15 and MyD88 in heterophils and monocyte-derived macrophages stimulated with Eimeria tenella in vitro. Exp Parasitol 133:427-433. https://doi.org/10.1016/j. exppara.2013.01.013

Publisher's note Springer Nature remains neutral with regard to jurisdictional claims in published maps and institutional affiliations. 